\title{
Effect of one-month treatment with methylphenidate on salivary cortisol level of attention deficit hyperactivity disorder children
}

Esraa Yahia Kholif* D, Salwa Mohamed Awad, Nasr Mohamed Attia, Ziyad Essam Tawhid and Mahmoud Elwasify

\begin{abstract}
Background: Attention deficit hyperactivity disorder (ADHD) is one of the most common neuropsychological disorders that affects children, adolescents, and adults. Dysfunction of the hypothalamic-pituitary-adrenal axis (HPA) has been implicated in its pathophysiology. So, this study aimed to compare the salivary cortisol level between ADHD children and healthy children. It also compared the salivary cortisol level of ADHD children before treatment and 1 month after treatment with methylphenidate.

Results: This study showed that there was a statistically significant decrease in salivary cortisol level of the ADHD group, specifically hyperactive/impulsive subtype compared to the control group. Furthermore, it showed a statistically significant increase of salivary cortisol level after treatment compared to before treatment in the ADHD group.

Conclusions: HPA axis dysfunction may be related to the pathophysiology of ADHD, specifically hyperactive/impulsive subtype. Methylphenidate positively affects the HPA axis of ADHD children.
\end{abstract}

Keywords: ADHD, Salivary cortisol, HPA, Methylphenidate

\section{Background}

Attention deficit hyperactivity disorder (ADHD) is one of the most common neuropsychological disorders that emerges in early childhood. The main characteristics of ADHD are impulsivity, hyperactivity, and inattention. There are three subtypes of ADHD which are predominantly hyperactive/impulsive, predominantly inattentive, and combined subtypes. The most common widespread subtypes are combined subtype then predominantly inattentive subtype. ADHD is diagnosed using the Diagnostic and Statistical Manual of Mental Disorders (DSM-V) criteria. Methylphenidate is one of the most effective stimulants used for its treatment for children aged 6 years and older [1-7].

\footnotetext{
* Correspondence: esraa.kholif@yahoo.com
Mansoura University, Mansoura, Egypt

* Correspondence: esraa.kholif@yahoo.con
Mansoura University, Mansoura, Egypt
}

(c) The Author(s). 2021 Open Access This article is licensed under a Creative Commons Attribution 4.0 International License, which permits use, sharing, adaptation, distribution and reproduction in any medium or format, as long as you give appropriate credit to the original author(s) and the source, provide a link to the Creative Commons licence, and indicate if changes were made. The images or other third party material in this article are included in the article's Creative Commons licence, unless indicated otherwise in a credit line to the material. If material is not included in the article's Creative Commons licence and your intended use is not permitted by statutory regulation or exceeds the permitted use, you will need to obtain permission directly from the copyright holder. To view a copy of this licence, visit http://creativecommons.org/licenses/by/4.0/. HPA axis function has been assessed by measuring the cortisol level in body fluids (saliva, blood, and urine) $[8,9]$.

S.Himani et al. [10] reported that attention deficit hyperactivity disorder/oppositional defiant disorder (ADHD/ ODD) children not taking stimulant drugs had lower salivary cortisol level than normal controls supporting the possibility of dysfunction of the HPA axis of these children. William et al. [11] demonstrated that severe inattentive ADHD children showed a decline in salivary cortisol after inducing social and cognitive stressors. However, moderate inattentive ADHD children and normal children showed an increase in cortisol concluding that the severe inattentive group showed dysregulation of the HPA axis. (HPA) axis which has a very critical role in controlling neurotransmitters of the central nervous system and behaviors such as attention, emotion, memory, and learning has been implicated in the pathophysiology of ADHD.

Dysfunction of the hypothalamic-pituitary-adrenal . . . . 
Liang-Jen et al. [12] showed that salivary dehydroepiandrosterone (DHEA) and DHEA/cortisol ratio were lower in ADHD children than in controls and correlated to continuous performance test (CPT). However, the level of salivary cortisol did not change between ADHD and normal children. Liang-Jen et al. [13] determined the salivary cortisol levels in ADHD children medicated with methylphenidate and healthy children over 6 months. This study showed that salivary cortisol levels of ADHD children increased significantly after 1 month of methylphenidate treatment then decreased to an intermediate level. Furthermore, salivary cortisol levels had a significant positive correlation with neuropsychological performance over the 6-month treatment period.

Alkhodier et al. [14] reported that medicated ADHD children did not show any significant change in salivary cortisol, buffering capacity, flow rate, $\mathrm{pH}$, and level of total salivary protein in comparison with non-medicated ADHD and healthy children. There were few studies concerning ADHD, salivary cortisol, and methylphenidate, and their results were controversial. So, this study was conducted to compare the salivary cortisol levels between ADHD children and healthy children and to determine the effect of 1-month treatment with methylphenidate on the salivary cortisol level of ADHD children.

\section{Aim}

The aims were to compare the salivary cortisol levels between ADHD children and healthy children and to compare the salivary cortisol level of ADHD children before treatment and 1 month after treatment with methylphenidate.

\section{Methods}

\section{Participants}

This prospective case-control study was completed in the period from December 2018 to December 2019. The study protocol approval was obtained from the Ethical Committee of Faculty of Dentistry, Mansoura University. Also, written informed consents were obtained from parents of both ADHD children and healthy children for participation in the study. It was conducted on 120 children. In group 1, 60 ADHD children (23 predominantly inattentive, 17 predominantly hyperactive/impulsive, 20 combined) were selected from the child and adolescent outpatient clinic of the Psychiatry Department, Mansoura University Hospital after being diagnosed by a consultant psychiatrist by semi-structured interview (mini Kid) using the Diagnostic and Statistical Manual of Mental Disorders (DSM5) [7] diagnostic criteria. The inclusion criteria included the following: (1) children aged 6 to 12 years; (2) non-medicated, newly diagnosed children; and (3) methylphenidate prescribed to children. The exclusion criteria included the following: (1) children receiving drugs of any chronic illness, (2) children with comorbid psychiatric disorders, and (3) non-methylphenidate-prescribed children. In group 2, sixty healthy children were selected randomly from primary schools in Mansoura city from grade 1 to grade 6, comparable in age, sex, socio-economic status, and parents' smoking habits to group 1 . The inclusion criterion included the following: children aged 6 to 12 years. The exclusion criteria included the following: (1) children receiving drugs of any chronic illness and (2) children with a history of psychiatric disorders.

\section{Clinical measurements}

Unstimulated salivary samples $[15,16]$ were collected in the morning (8:30-9:30 a.m) for both groups. Saliva was collected from ADHD children twice initially before treatment and later after 1 month of treatment with methylphenidate (prescribed dose $=0.5-2 \mathrm{mg} / \mathrm{kg} /$ day) and once from healthy children. For ADHD children, the first samples were taken from 60 children, and the second samples were taken from 45 children because of drop-out cases (Fig. 1). Children were instructed not to eat or drink, not to chew any chewing gums, not to brush their teeth, and not to use any mouthwashes $1 \mathrm{~h}$ before saliva collection. Children were allowed to sit in an upright position on the chair and relax for a few minutes. Saliva was collected by asking the child to expectorate into a clean plastic cup, then a plastic syringe with a removed piston was used to aspirate $4-5 \mathrm{ml}$ saliva from the plastic cup. Then, the saliva was transferred to a sterile container to be stored at $-20^{\circ} \mathrm{C}$. After that, salivary samples were sent to the laboratory for cortisol analysis using the IMMUNOSPEC ELISA kit (IMMUNOSPEC CORPORATION, Catalog No. E18-188, Canada). Cortisol analysis was done in Clinical Immunology Unit, Clinical Pathology Department, Mansoura Faculty of Medicine.

\section{Statistical analysis}

The calculated sample size of this study was 44 children for each group at $95 \%$ confidence level, $80 \%$ power, and $5 \%$ level of significance via this formula:

$$
\begin{aligned}
& N=(\mathrm{Z} 1-\alpha / 2+\mathrm{Z} 1-\beta)^{2} \sigma 1 \times \sigma 2 / \delta^{2} \\
& \mathrm{Z} 1-\alpha / 2=1.96 \\
& \mathrm{Z} 1-\beta=0.842
\end{aligned}
$$$$
\text { Mean cortisol level in ADHD }=10.17
$$

Mean cortisol level in normal group $=14.23$

$\sigma 1=\mathrm{SD}$ in $\mathrm{ADHD}=7.35$

$\sigma 2=\mathrm{SD}$ in the normal group $=12.76$

$\delta=$ expected difference to be detected between the studied groups (4.0)

$\alpha=$ level of acceptability of a false positive result (level of significance $=0.05$ )

$\beta=$ level of acceptability of a false-negative result $(0.20)$

$1-\beta=$ power $(0.80)$ 


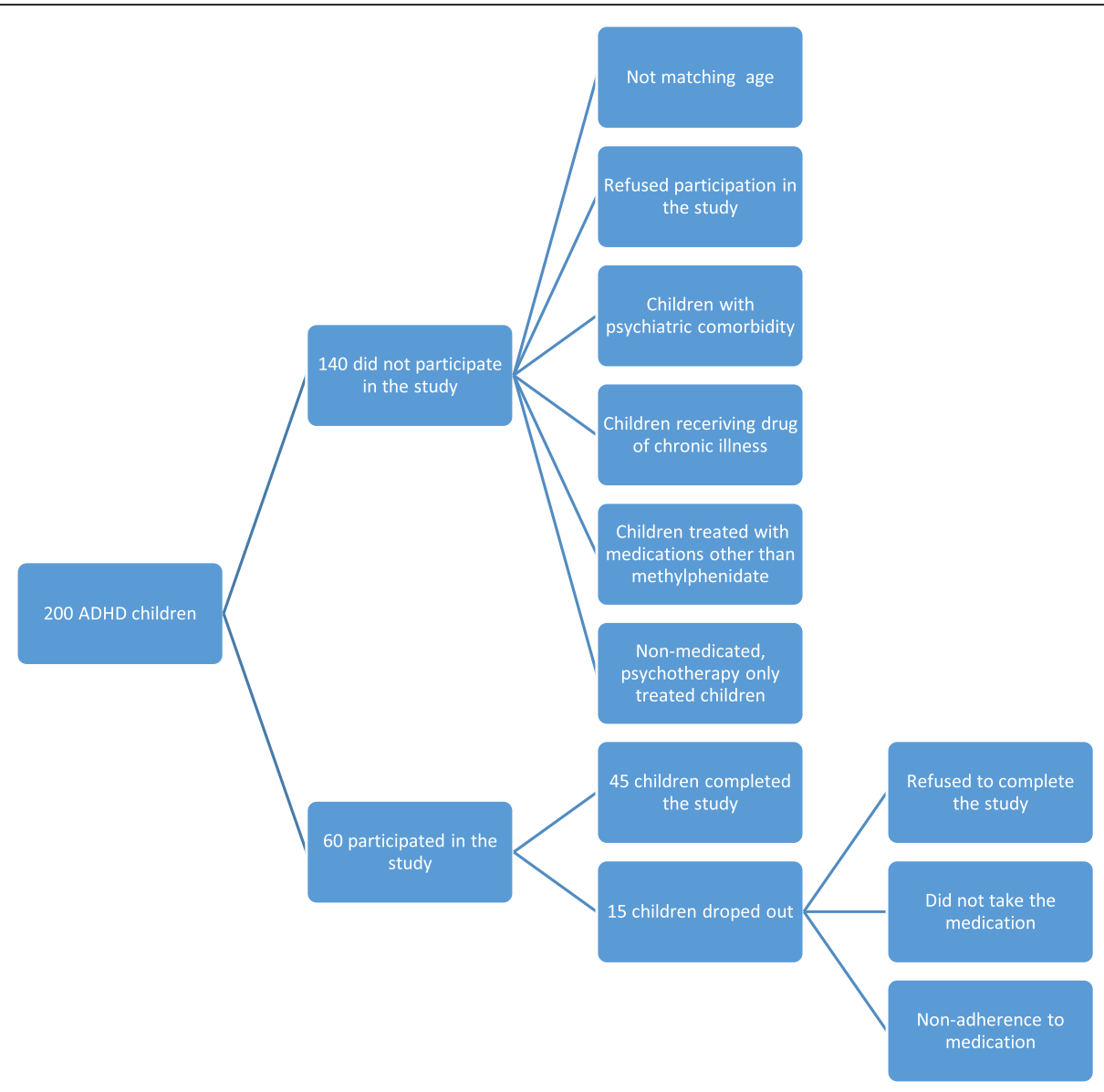

Fig. 1 Flow chart presenting ADHD children selection

The sample size was increased to 60 children for each group for compensation of incomplete data and for increasing the study power.

Statistical Package of Social Science (SPSS) program for Windows (standard version 24) was used for data analysis. The normality of data was tested using the onesample Kolmogorov-Smirnov test. Qualitative data were described using number and percent. The association between categorical variables was tested using the chisquare test. Continuous variables were presented as mean \pm SD (standard deviation) for parametric data and median (min-max) for non-parametric data. The two groups were compared with the Student $t$ test for parametric data and the Mann-Whitney test for nonparametric data while paired non-parametric data was compared by Wilcoxon signed rank test. The results were considered to be significant when the probability value equal or less than $5 \%(p \leq 0.05)$.

\section{Results}

Sixty ADHD children (23 predominantly inattentive, 17 predominantly hyperactive/impulsive, 20 combined) and sixty healthy children were included in the study, 46 males and 14 females in each group and aged 6 to 12 years. There were no statistically significant differences between the two groups regarding sociodemographic data (age, sex, education, occupation, income, parents' smoking habits) (Table 1).

Comparing the salivary cortisol level between the ADHD group before treatment, its subtypes, and the control group revealed that the ADHD group and hyperactive/impulsive subtype had a statistically significantly lower salivary cortisol level compared to the control group ( $p=0.038,0.036$, respectively). However, there were no significant differences between the inattentive subtype and the control groups, between the combined subtype and the control groups, and between the 3 ADHD subtypes regarding salivary cortisol level (Table 2).

Comparing the salivary cortisol levels in the ADHD group before treatment and 1 month after treatment with methylphenidate revealed a statistically significant increase in salivary cortisol levels after treatment compared to before treatment $(p=0.035)$. The inattentive subtype, hyperactive/impulsive subtype, and combined subtype showed no significant difference after treatment compared to before treatment (Table 3). 
Table 1 Sociodemographic data of the ADHD and control groups

\begin{tabular}{|c|c|c|c|}
\hline Variables & ADHD group, $n=60(\%)$ & Control group, $n=60(\%)$ & Test of significance, $p$ value \\
\hline \multicolumn{4}{|l|}{ Age, years } \\
\hline Mean \pm SD & $8.65 \pm 2.11$ & $8.68 \pm 2.05$ & $t=0.086, p=0.932$ \\
\hline Min-max & $6-12$ & $6-12$ & \\
\hline \multicolumn{4}{|l|}{ Sex } \\
\hline Male & $46(76.7 \%)$ & $46(76.7 \%)$ & $x^{2}=0, p=1.0$ \\
\hline Female & $14(23.3 \%)$ & $14(23.3 \%)$ & \\
\hline \multicolumn{4}{|l|}{ Education } \\
\hline High & $22(36.7 \%)$ & $21(35 \%)$ & $x^{2}=0.325, p=0.850$ \\
\hline Moderate & $32(53.3 \%)$ & $31(51.7 \%)$ & \\
\hline Low & $6(10 \%)$ & $8(13.3 \%)$ & \\
\hline \multicolumn{4}{|l|}{ Occupation } \\
\hline Professionals & $10(16.7 \%)$ & $13(21.7 \%)$ & $x^{2}=0.693, p=0.707$ \\
\hline Office worker & $31(51.7 \%)$ & $27(45.0 \%)$ & \\
\hline Manual worker & $19(31.7 \%)$ & $20(33.3 \%)$ & \\
\hline \multicolumn{4}{|l|}{ Income } \\
\hline High & $13(21.7 \%)$ & $12(20.0 \%)$ & $x^{2}=157, p=0.925$ \\
\hline Moderate & $39(65.0 \%)$ & $41(68.3 \%)$ & \\
\hline Low & $8(13.3 \%)$ & $7(11.7 \%)$ & \\
\hline Parents' smoking habit & $23(38.3 \%)$ & $24(40 \%)$ & $x^{2}=0.035, p=0.852$ \\
\hline
\end{tabular}

Comparing the salivary cortisol levels between the ADHD group after treatment, its subtypes and the control group revealed that the ADHD group, inattentive subtype, hyperactive/impulsive subtype, and combined subtype had no statistically significant difference in salivary cortisol level compared to the control group (Table 4).

\section{Discussion}

This study aimed to compare the salivary cortisol level of ADHD children versus healthy children. Also, it aimed to compare salivary cortisol level of ADHD children before treatment and one month after treatment with methylphenidate. At the present study, sixty children were selected for both groups aged from 6 to 12 years in agreement with the study of Liang-Jen et al. [13] concerning age and because methylphenidate was recommended to be prescribed for children aged 6 years and older [1].

ADHD children who participated in the study were non-medicated, newly diagnosed, methylphenidateprescribed and free from chronic diseases and psychiatric disorders other than ADHD, and control children were healthy and free from chronic diseases and psychiatric disorders. These criteria were selected to attribute any difference before treatment to the presence of the disorder itself, not to the effect of the medication, and also to determine the effect of methylphenidate on salivary cortisol level of ADHD children.
Unstimulated salivary samples were collected because salivary samples are less invasive and not as painful as blood samples. Also, unstimulated salivary samples are more comfortable, less invasive, and more accurate than stimulated salivary samples $[15,16]$. Salivary samples were stored at $-20{ }^{\circ} \mathrm{C}$ according to the manufacturer's instructions. Then, they were sent for laboratory analysis using the ELISA kit.

In this study, $76.7 \%$ of the examined children were males, and 23.3\%were females in each group. ADHD is more common in males than in females. Many previous studies reported male to female ratio in ADHD as 2.5:1 [4], 8:1 [17], and 5:1 [18].

Concerning salivary cortisol level, this study demonstrated that the ADHD group had a statistically significant decrease in salivary cortisol level than the control group in agreement with the studies of Enrique et al. [19], Catia et al. [20], and Johan et al. [21].

The study of S.Himani et al. [10] concluded that salivary cortisol level was lower in ADHD/ODD children not taking stimulant drugs than in normal controls. Also, the study of Liang et al. [22] reported that ADHD children had a significantly lower plasma cortisol level compared to the control group. These results supported the possibility of dysfunction of the HPA axis in ADHD children.

On the contrary, the results of the present study disagreed with the results of the studies of Liang-gen et al. 


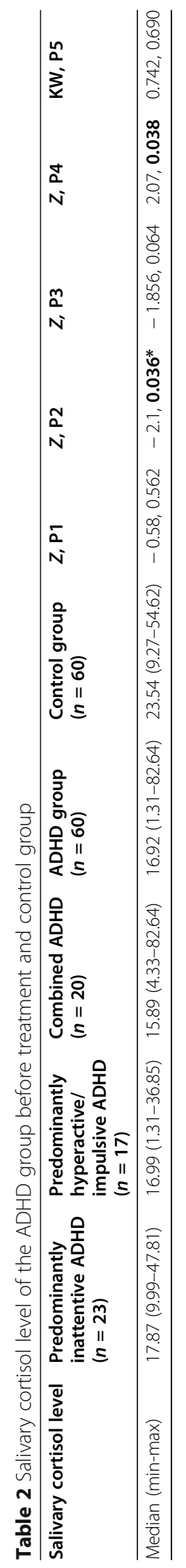


Table 3 Salivary cortisol level of the ADHD group before treatment and 1 month after treatment with methylphenidate

\begin{tabular}{lllll}
\hline Salivary cortisol level & $\begin{array}{l}\text { Predominantly inattentive } \\
\text { ADHD }(\boldsymbol{n}=\mathbf{1 7})\end{array}$ & $\begin{array}{l}\text { Predominantly hyperactive/ } \\
\text { impulsive ADHD }(\boldsymbol{n}=\mathbf{1 2})\end{array}$ & Combined ADHD $(\boldsymbol{n}=\mathbf{1 6})$ & ADHD group ( $\boldsymbol{n}=\mathbf{4 5})$ \\
\hline Before treatment & $15.98(9.99-44.40)$ & $16.95(1.31-38.85)$ & $15.90(4.33-82.64)$ & $16.04(1.31-82.64)$ \\
After treatment & $25.74(9.67-78.13)$ & $27.75(2.45-47.41)$ & $22.42(1.18-99.34)$ & $25.74(1.18-99.34)$ \\
Wilcoxon signed test & 1.82 & 1.88 & 0.362 & 2.11 \\
$p$ value & 0.068 & 0.06 & 0.717 & $\mathbf{0 . 0 3 5 ^ { * }}$ \\
\hline
\end{tabular}

[12] and Alkhodier et al. [14] which showed no difference in salivary cortisol level between the ADHD and control groups. This difference might be due to the differences in sample size, the study of Alkhodier et al. [14], and the number of the examined non-medicated ADHD children and normal children was 17. Also, the study of Hanna et al. [23] revealed no significant difference between the ADHD and control groups regarding salivary cortisol levels, but $37.1 \%$ of ADHD children were on stimulant medication.

The results of comparing salivary cortisol level between the ADHD subtypes and the control group revealed that the hyperactive/impulsive (HI) ADHD subtype had a statistically significant decrease of salivary cortisol level compared to the control group in agreement with the study of Blomqvist et al. [24] which confirmed that HI ADHD had a significantly reduced salivary cortisol level $30 \mathrm{~min}$ after awaking compared to the control group. Also, this study showed no statistically significant difference in salivary cortisol level between the inattentive (I) ADHD subtype and the control group in agreement with the study of William et al. [11] which showed no significant difference between the moderately inattentive, threshold inattentive, and control groups regarding salivary cortisol level at baseline.

The present study reported no statistically significant difference between the combined (C) ADHD subtype and the control groups and between the 3 ADHD subtypes regarding salivary cortisol. The descending order of salivary cortisol level between the 3 ADHD subtypes was ADHD-HI then ADHD-C then ADHD-I ( $p=0.036$, $0.064,0.562$, respectively). However, the study of Liang et al. [22] concluded that the 3ADHD subtypes had significantly lower cortisol levels than the control group, and the descending order of the cortisol level in the plasma was in ADHD-HI, ADHD-I, and then ADHD-C, and there was no significant difference between the ADHD-I and ADHD-C groups. These results support the possibility that HPA axis dysfunction in ADHD children is related to hyperactivity more than inattention.

At the present study, salivary cortisol level was measured for 60 ADHD children before treatment and 45 ADHD children after treatment at the second visit because of drop out cases. At the study of Liang-Jen et al.
[13], the salivary cortisol was measured for 50 ADHD children before treatment and 42 ADHD children after treatment at the second visit. At the present study, the dose of methylphenidate was prescribed for ADHD children according to age, weight, and severity of symptoms in agreement with clinical guidelines for prescribing methylphenidate for ADHD children.

Concerning the effect of 1-month treatment with methylphenidate on salivary cortisol level of ADHD children, the present study showed that salivary cortisol level of ADHD children had a statistically significant increase after 1-month treatment with methylphenidate compared to before treatment in agreement with Liang-Jen et al. [13] supporting the possibility that methylphenidate intake positively affects the function of HPA axis in ADHD children. The inattentive subtype, hyperactive/impulsive subtype, and combined subtype showed no significant difference after treatment compared to before treatment ( $p=0.068,0.06,0.717$, respectively). Also, the study of Moon-Soo et al. [25] revealed that plasma cortisol levels had not changed significantly after a 12-week treatment with methylphenidate or bupropion in combined ADHD children.

The result of this study demonstrated that there was no statistically significant difference in salivary cortisol level between the ADHD and control groups, between the ADHD-HI, ADHD-I, ADHD-C, and control groups and between the 3 ADHD subtypes after 1-month treatment with methylphenidate. The study of S.Himani et al. [10] showed that ADHD/ODD patient who had taken stimulants either methylphenidate or D-amphetamine had salivary cortisol levels that are not significantly different from normal individuals. Also, the study of Alkhodier et al. [14] showed that salivary cortisol levels did not differ significantly between the control group and the medicated ADHD group.

\section{Limitations}

The following are the limitations of this study: shortterm evaluation of the effect of methylphenidate, single-center study, and the small sample size of the ADHD subtypes. 


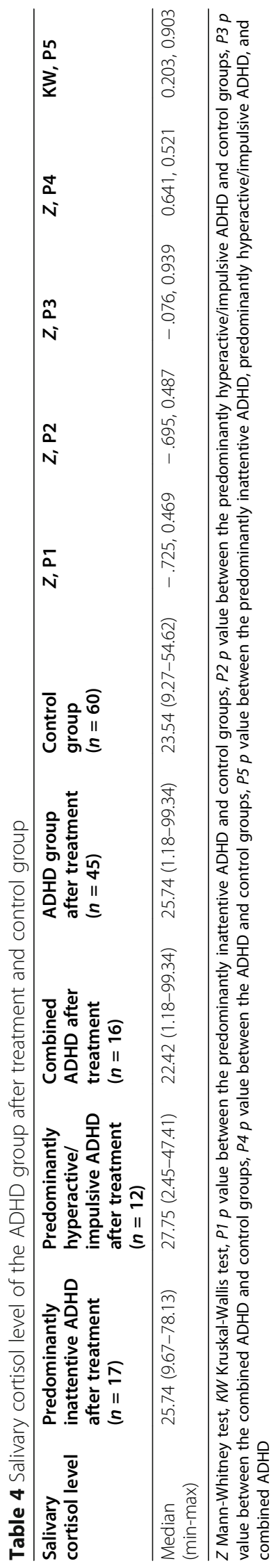




\section{Conclusions}

HPA axis dysfunction may be related to the pathophysiology of ADHD, specifically hyperactive/impulsive subtype. Methylphenidate positively affects the HPA axis of ADHD children.

\section{Recommendations}

Further studies are recommended to be conducted with a longer follow-up time than 1 month to determine if the effect of methylphenidate in salivary cortisol is temporary or permanent, steady, or changeable. Further studies are recommended to be conducted on ADHD children medicated with non-stimulant drugs (e.g., atomoxetine). Further studies are recommended to be conducted on ADHD subtypes with a larger sample size.

\section{Abbreviations}

ADHD: Attention deficit hyperactivity disorder; HPA: Hypothalamic-pituitaryadrenal; DSM5: Diagnostic and Statistical Manual of Mental Disorders fifth edition; ODD: Oppositional defiant disorder; DHEA: Dehydroepiandrosterone; CPT: Continuous performance test; ADHD-HI: Hyperactive/impulsive attention deficit hyperactivity disorder; ADHD-C: Combined attention deficit hyperactivity disorder; ADHD-I: Inattentive attention deficit hyperactivity disorder

\section{Acknowledgements}

No acknowledgement to be included.

\section{Authors' contributions}

E.K performed the practical part of the study, wrote the paper, and ensured that all authors approved the paper before submission. M.W diagnosed the ADHD children, contributed to the idea and design of the study, revised the paper, and approved it. S.A contributed to the idea of the study, revised the paper, and approved it. N.A contributed to the idea of the study, revised the paper, and approved it. Z.T supervised the cortisol analysis work, revised the paper, and approved it. The authors read and approved the final manuscript.

\section{Funding}

No funding obtained for the present research.

\section{Availability of data and materials}

Data is available upon request.

\section{Ethics approval and consent to participate}

The study protocol approval was obtained from the Ethical Committee of Faculty of Dentistry, Mansoura University (Code No. 02030718). Also, written informed consents were obtained from parents of both ADHD children and healthy children for participation in the study after informing them about the aim and the method of the study.

\section{Consent for publication}

As a corresponding author, I confirm that the paper has been revised and approved for submission by all the named authors.

\section{Competing interests}

There is no conflict of interest associated with this publication.

Received: 24 December 2020 Accepted: 15 February 2021 Published online: 02 March 2021

\section{References}

1. Association A (1994) Diagnostic and statistical manual of mental disorders, 4th edn. Am Psych Assoc, Washington DC, pp 6294-6304

2. Breton J, Bergeron L, Valla J, Berthiaume C, Gaudet N, Lambert J, Lépine S (1999) Quebec child mental health survey: prevalence of DSM-III-R mental health disorders. J Child Psychol Psych. 40:375-384

3. Chae $P$, Jung $H$, Noh K (2001) Attention deficit hyperactivity disorder in Korean juvenile delinquents. Adol. 36:707
4. No Authors Listed $(2003,2005)$ Control CFD and prevention, mental health in the United States. Prevalence of diagnosis and medication treatment for attention-deficit/hyperactivity disorder-United States. Morb and mort week repo.54:842.

5. Parker $H$ (2005) The ADHD handbook for schools: effective strategies for identifying and teaching students with attention-deficit/hyperactivity disorder. Special Press/ADD Warehouse, vol 43, p 48

6. Moher D, Varisco S, Rossettini G, Testa M (2009) Preferred Reporting Items for Systematic Reviews and Meta-Analyses: the PRISMA statement. Ann of intern med.151:264-326.

7. The Diagnostic and Statistical Manual of Mental Disorders, 5th edition $\left(\mathrm{DSM}-5^{\mathrm{TM}}\right)$ (2015). Am Psych Assoc.19:656

8. Talge N, Neal C, Glover V (2007) Antenatal maternal stress and long-term effects on child neurodevelopment: how and why? J Child Psychol Psych. 48:245-261

9. Hellhammer D, Wust S, Kudielka B (2009) Salivary cortisol as a biomarker in stress research. Psychoneuroendocrinol. 34:163-171

10. Himani SK, Frank Z, Sheila L (2002) Reduced salivary cortisol in children with comorbid attention deficit hyperactivity disorder and oppositional defiant disorder. Neuroendocrinol 23:45-48

11. William T, Randazzo SD, Elizabeth J, Susman (2008) The stress response in adolescents with inattentive type ADHD symptoms. Child Psych Hum Dev. 39:27-38

12. Liang-Jen W, Yu-Shu H, Chen-Cheng H, Yuan-Lin C, Chin-Ching W, Zong-Yi S, Chin-Ken C (2011) Salivary dehydroepiandrosterone, but not cortisol, is associated with attention deficit hyperactivity disorder. World J Biol Psych. 12:99-109

13. Liang-Jen W, Yu-Shu H, Cheng-Cheng H, Chih-Ken C (2017) The trend in morning levels of salivary cortisol in children with ADHD during six months of methylphenidate treatment. J Att Dis. 21:254-261

14. Alkhodier H, Wang H, Sun H, Zhong W, Cappelli D, Liu J, Maria-Jose C, Yeh C (2018) Saliva and oral health in attention deficit hyperactivity disorder. J Oral Med. 2:1-2

15. Heintz $\cup$ (1984) Secretion rate, buffer effect, number of lactobacilli and streptococcus mutans of whole saliva of cigarette smokers and nonsmokers. Scand J Dent Res .92:294-301.

16. Sullivan OE, Curzon M (2000) Salivary factors affecting dental erosion in children. Caries Res 34:82-87

17. Hidas A (2013) Salivary bacteria and oral health status in medicated and non-medicated children and adolescents with attention deficit hyperactivity disorder (ADHD). Clin Oral Investig. 17:1863-1867

18. Sharif F, Zarei S, Shooshtari A, Vossoughi M (2015) Approach on mental health of the mothers of the children with attention deficit hyperactivity disorder. Iran J Pediatr 25:474

19. Enrique F, Victoria T, Antonio C, Encarnación M, Milagros E (2009) Salivary cortisol response to a psychosocial stressor on children diagnosed with attention-deficit/hyperactivity disorder: differences between diagnostic subtypes. Span J Psychol 12:707-714

20. Catia S, Cristian B, Stephen V, Massimo G (2012) Biomarkers and attentiondeficit/hyperactivity disorder: a systematic review and meta-analyses. J Am Acad Child Adol Psych 51:10

21. Johan I, Kent W, Fred N, Åsa H, Frank L (2012) Cortisol levels in children with attention-deficit/hyperactivity disorder. J Psych Res 46:1398-1405

22. Liang M, Yan-Hui C, Hui C, Yan-Yan L, Yan-Xia W (2011) The function of hypothalamus-pituitary-adrenal axis in children with ADHD. Brain Res 1368: 159-162

23. Hanna C, Robert D, Lamprini P, Berthold P, Edmund J (2010) Does the cortisol response to stress mediate the link between expressed emotion and oppositional behavior in attention-deficit/hyperactivity-disorder (ADHD)? Behavioral and Brain Functions. 6:45

24. Blomqvist M, Holmberg K, Lindblad F, Fernell E, Ek U, Dahllo"f G (2007) Salivary cortisol levels and dental anxiety in children with attention deficit hyperactivity disorder. Eur J Oral Sci. 115: 1-6.

25. Moon-Soo L, Jae-Won Y, Young-Hoon K, Changsu H, Seung-Hyun K, MinSoo L, Sook-Haeng J, In-Kwa J (2008) Effects of methylphenidate and bupropion on DHEA-S and cortisol plasma levels in attention-deficit hyperactivity disorder. Child Psych Hum Dev. 39:201-209

\section{Publisher's Note}

Springer Nature remains neutral with regard to jurisdictional claims in published maps and institutional affiliations. 